\title{
High-efficiency absorber for damping transverse wakefields
}

\author{
A. Novokhatski, ${ }^{*}$ J. Seeman, and S. Weathersby \\ SLAC, Stanford University, 2575 Sand Hill Road, Menlo Park, California 94025, USA
}

(Received 5 February 2007; published 26 April 2007)

\begin{abstract}
Transverse wakefields generated by intense beams may propagate long distances in a vacuum chamber and dissipate power in different shielded elements such as bellows, vacuum valves, or vacuum pumps. Induced heating in these elements may be high enough to deteriorate vacuum conditions. We have developed a broadband water-cooled bellows absorber to capture and damp these harmful transverse fields without impacting the longitudinal beam impedance. Experimental results at the PEP-II SLAC B-factory demonstrate a high efficiency for this device. This absorber may be useful in super B-factories, the International Linear Collider, the Large Hadron Collider, or synchrotron light sources.
\end{abstract}

DOI: 10.1103/PhysRevSTAB.10.042003

PACS numbers: 29.20.Dh, 42.25.Dd, 84.40.- $\mathrm{x}$

\section{INTRODUCTION}

The SLAC PEP-II asymmetric B-factory collides 3.0 A of $3 \mathrm{GeV}$ positrons on $1.9 \mathrm{~A}$ of $9 \mathrm{GeV}$ electrons in 1700 bunches. The B-factory consists of a low energy positron storage ring (LER) situated above a high energy electron storage ring (HER). The rings intersect at an interaction point (IP) within the $B A B A R$ detector sustaining a luminosity of $1.2 \times 10^{34} \mathrm{~cm}^{-2} \mathrm{~s}^{-1}$ [1]. A very low longitudinal impedance vacuum chamber allowed us to achieve extremely high currents.

However, we found elevated temperature on shielded bellows and vacuum valves around the rings. Longitudinal wakefields have a very small coupling with shielded bellows cavities, but transverse fields can easily penetrate through the fingers and excite different resonant modes. As transverse fields may propagate long distances in the aluminum chamber, the wakefield source can be far away from the bellows position. Of particular concern was anomalously high temperature observed on a first arc bellows, located 15 meters downstream of a fixed vertical beam collimator. Thermocouples mounted on the exterior of the bellows within the convolutions were registering a temperature rise of $80^{\circ} \mathrm{F}$, reaching $220^{\circ} \mathrm{F}$ at nominal LER currents of $2500 \mathrm{~mA}$ even after the installation of cooling fans. Internally, sensitive bellows fingers can reach much higher temperatures. In this region the power in the wakefields was high enough to destroy the feedthrough for the titanium sublimation pump (TSP). To solve this problem we installed an arc absorber directly in the antechamber part of the pumping section to capture these wakefields [2]. The power loss measured in the absorber reaches $1200 \mathrm{~W}$ for a positron beam current of $2.4 \mathrm{~A}$. This arc absorber was useful in locating the source of wakefields. Steering the beam at the vertical collimator upstream of the absorber resulted in the significant power change in the absorber. There was no power change when we tried to steer the beam at the horizontal collimator. Thus, only vertically

*Electronic address: novo@slac.stanford.edu polarized fields propagate in this pumping chamber while the temperature rise in the upstream arc bellows correlates with beam position at both collimators [3].

The requirement for higher luminosity entails higher ring currents at the 3-4 A levels as well as shorter bunch rms length of $0.8 \mathrm{~cm}$, both of which contribute to more bellows heating. To solve this problem we have used our understanding of the wakefield nature to design a special absorber.

In the absorbers, which were developed to damp higher order modes (HOM) near rf cavities, the absorbing material is usually exposed to the beam. This causes additional beam energy losses due to diffraction of the beam field and Cherenkov radiation, when the absorbing material has a noticeable permittivity or magnetic permeability [4-6]. The design of a wing-type HOM damper [7] needs too much space in the ring. There are two slots of length $600 \mathrm{~mm}$. It was designed to damp only one polarization of dipolelike modes and consequently has less efficiency for quadrupolelike modes. In our case we had only $70 \mathrm{~mm}$ available for slots. We optimized the geometry of absorbing tiles, the number of slots, and their width to avoid additional losses from the longitudinal fields while efficiently damping all transverse fields. The proposal for the absorber was presented at PAC'2005 [3,8] and first results were presented at EPAC'2006 [9].

\section{COLLIMATOR WAKEFIELDS}

Besides measurement, we carry out numerical studies of wakefields excited near a collimator using the MAFIA code [10]. The shape of the PEP-II LER straight section collimator is shown in Fig. 1. It consists of a central flat protrusion inside a round chamber. The protrusion has tapers at both ends. The total length is $60.8 \mathrm{~cm}$. We did simulations for the vertical collimator, which has a $9 \mathrm{~mm}$ nominal offset from the collimator edge to the beam axis. We compute the loss factor of a $13 \mathrm{~mm}$ long Gaussian bunch passing the vertical collimator at different offsets in vertical and horizontal positions relative to the collimator. 


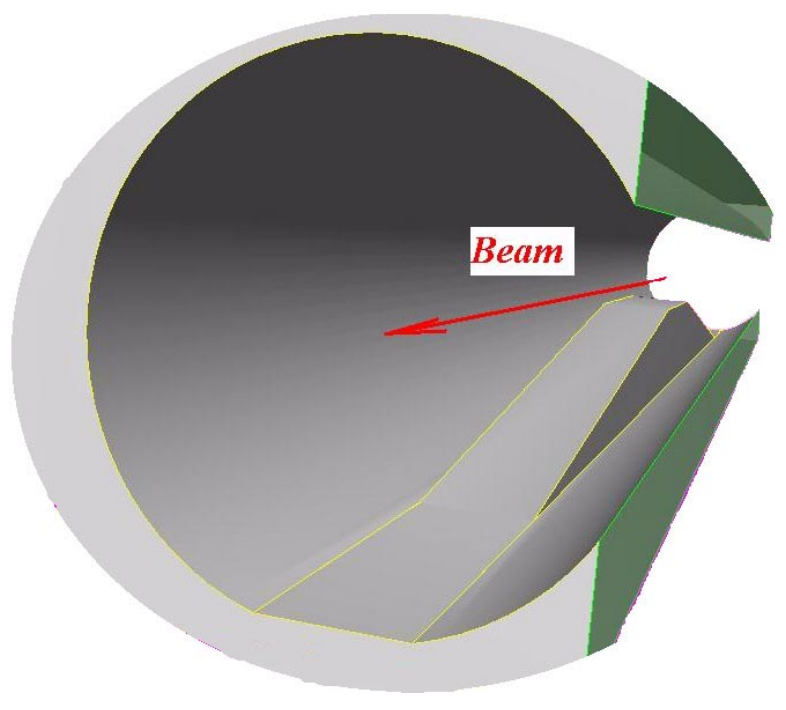

FIG. 1. (Color) PEP-II LER collimator.

These results, which are shown in Figs. 2 and 3, confirm our measurements. When we move the beam closer to the collimator edge in the vertical direction the arc absorber sees more power. When we move the beam in the horizontal direction, the power in the absorber did not change much.

Loss factor $k$, beam current $I$, and bunch spacing $\tau$ give the power generated by the beam in a frequency range above the cutoff frequency [11]

$$
P=k \tau I^{2}
$$

Note that there is a set of high $Q$ trapped collimator modes $[12,13]$, which may give additional heating to the collimator. Naturally these modes stay near the collimator and do not propagate in the ring. However, the loss factor for standing wave modes is small in comparison with the

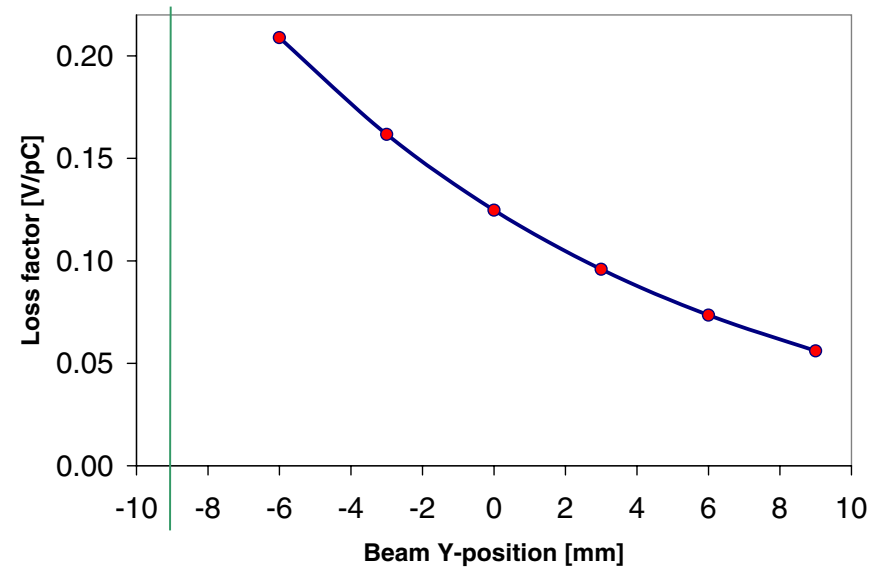

FIG. 2. (Color) Loss factor of a $13 \mathrm{~mm}$ long Gaussian bunch passing the vertical collimator at different offsets in the vertical position. The green line shows the collimator edge.

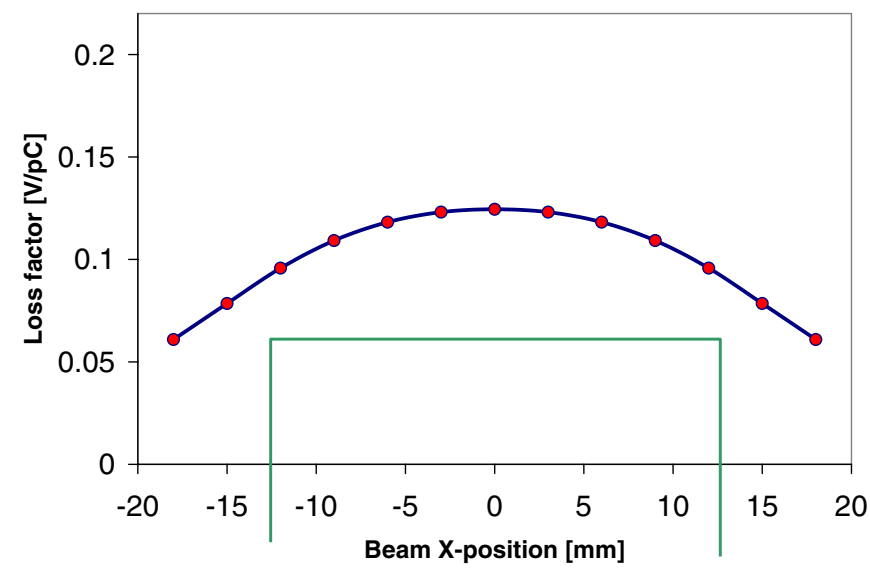

FIG. 3. (Color) Loss factor of a $13 \mathrm{~mm}$ long Gaussian bunch passing the vertical collimator at different offsets in the vertical position. The green line shows the collimator edge. Loss factor of a $13 \mathrm{~mm}$ long Gaussian bunch passing the vertical collimator at different offsets in the horizontal position. The green line shows the collimator edge.

loss factor for propagating modes. We can consider the total loss factor to be due to propagating waves.

For the current beam parameters: $I=2.4 \mathrm{~A}, \tau=4.2 \mathrm{~ns}$ (bunch spacing by 2), and $k=0.125 \mathrm{~V} / \mathrm{pC}$ (bunch length $\sigma=13 \mathrm{~mm}$ at nominal beam position at the collimator), this power is $3 \mathrm{~kW}$. This number looks to be reasonable. We measured captured power in the arc absorber of $1.2 \mathrm{~kW}$. The arc absorber is $15 \mathrm{~m}$ far away from the collimator, so some power is dissipated in the chamber wall. Some power goes to the bellows, a vacuum valve, or propagates to the next arc chambers. (We will get a better estimation of collimator wakefield power with a new bellows absorber.) Calculated bunch length dependence of the loss factor (Fig. 4) is well approximated with an inverse quadratic function. This is also in agreement with measurement of the power in the arc absorber, which is linear with $\mathrm{rf}$ voltage [2] and is equivalent to an inverse quadratic dependence on the bunch length.

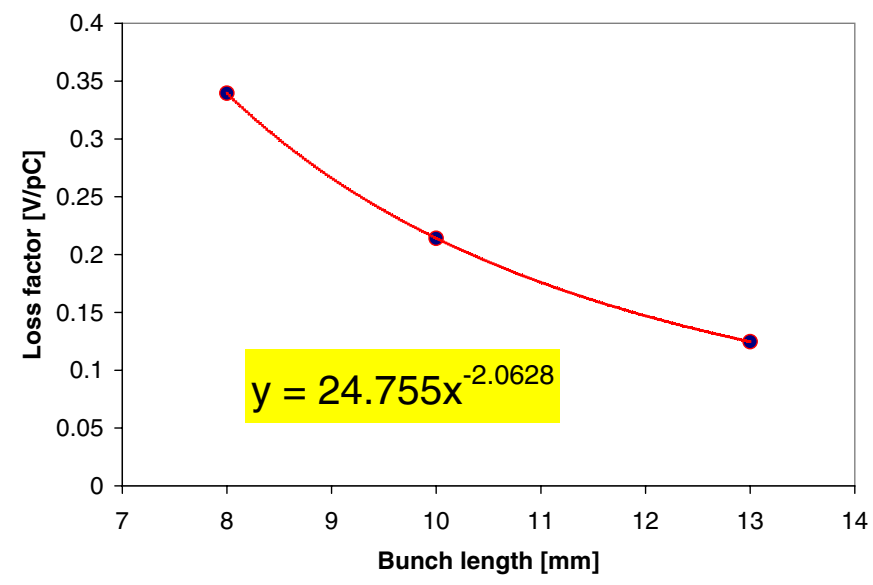

FIG. 4. (Color) Loss factor as a function of bunch length. 


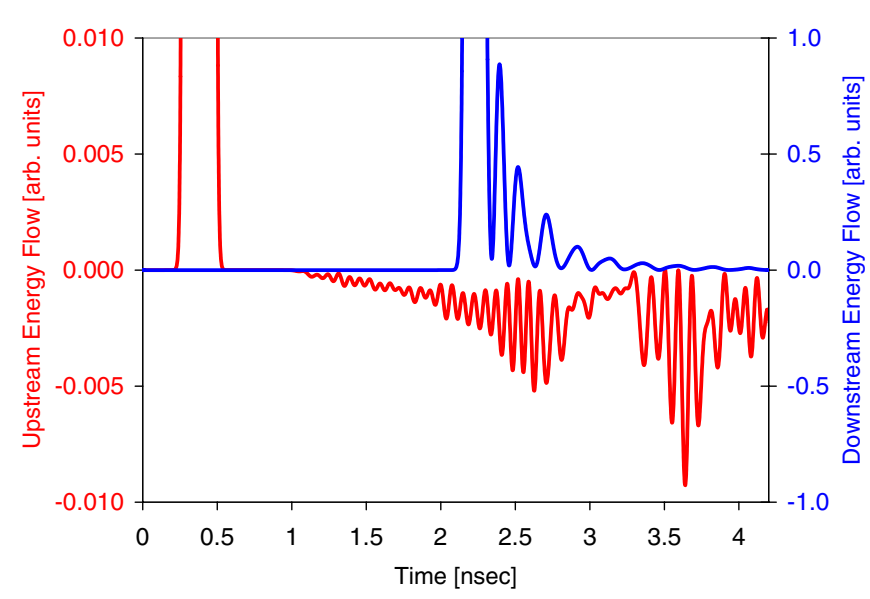

FIG. 5. (Color) Time dependence of the energy flow through the incoming (red) and outgoing (blue) chamber cross sections of the collimator. Scales are different by 100 times.

In simulations we found the direction of the wakefield energy flow to be the same as in experiment. Steering the beam at the collimator, we found the temperature change only in the bellows, which are downstream of the collimator. Figure 5 shows the calculated energy flow at two ends of the collimator. Energy flow was calculated as the integral of the Poynting vector over the chamber cross section. In Fig. 4 a positive energy flow indicates flow in the beam direction. Negative flow is in the upstream direction. The double peak shape of upstream energy flow originates at the two sloped faces of the collimator.

The calculated frequency spectrum of the collimator wakefield of a $13 \mathrm{~mm}$ long Gaussian bunch was also found to be similar to the spectrum measurement. A spectrum analyzer and antenna were installed in the tunnel at the

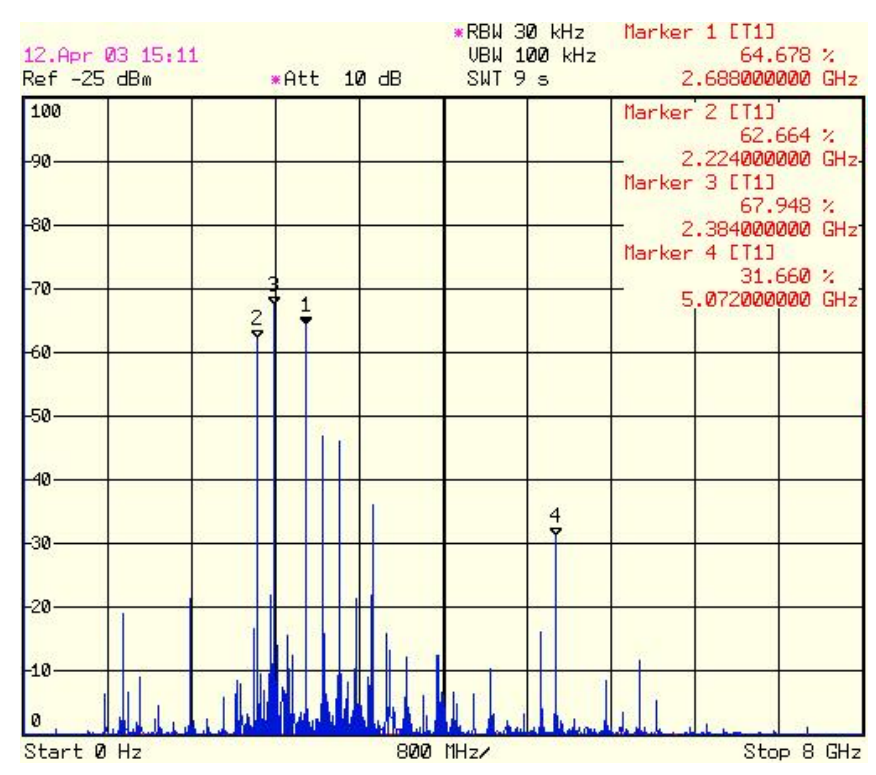

FIG. 6. (Color) Wake field spectrum measured in the PEP-II tunnel in a multibunch regime.

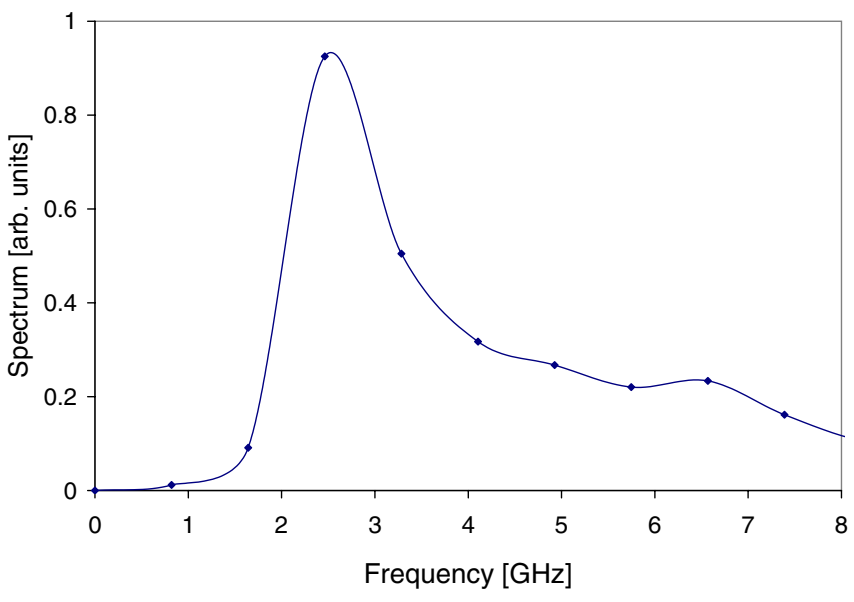

FIG. 7. (Color) Calculated spectrum of the collimator wakefield of a single bunch.

TSP feedthrough [2]. Figure 6 shows the measured spectrum in a multibunch regime. Figure 7 shows the calculated wakefield spectrum for a single bunch. Spectrum has a maximum in the frequency range of $2-3 \mathrm{GHz}$ (cutoff frequency for the $\mathrm{TE}_{11}$ mode is $1.99 \mathrm{GHz}$ ).

We also study the detailed structure of the wakefields. It was determined that collimator wakefields have mainly the form of dipole and quadrupole propagating modes. Figure 8 shows snapshots of electric field force lines downstream from the collimator during $1 \mathrm{~ns}$ after the passage of a bunch. They have a dipole and quadrupole field pattern. The dipole mode predominates. Based on these studies a device was designed to couple out and damp dipole, quadrupole, and higher modes while having little interaction with longitudinal (monopole) mode. The coupled mode power is concentrated and dissipated in water-cooled ceramic tiles.

\section{STRAIGHT SECTION BELLOWS ABSORBER}

We consider a device with coupling slots and a cavity containing high loss tangent ceramic tiles. We combine a shielded bellows and an absorber in one device: bellows absorber.

\section{A. Ceramic tiles characterization}

SLAC has extensive experience [14] in using Ceradyne Inc. [15] ceramic tiles as an effective absorbing material. We successfully used these tiles in the arc absorber [2]. The material of a tile (Ceralloy 13740Y) consists of aluminum nitride (AlN) with $40 \%$ silicon carbide (SiC). Although the company gives the table of dielectric properties of this material, we measured the relative permittivity and loss tangent at S-band frequency. We used the fact that the resonant frequency $f_{d}$ of a cavity depends on the relative permittivity $\epsilon$ of the material filling a cavity:

$$
f_{d}=f_{0} / \sqrt{\epsilon}
$$




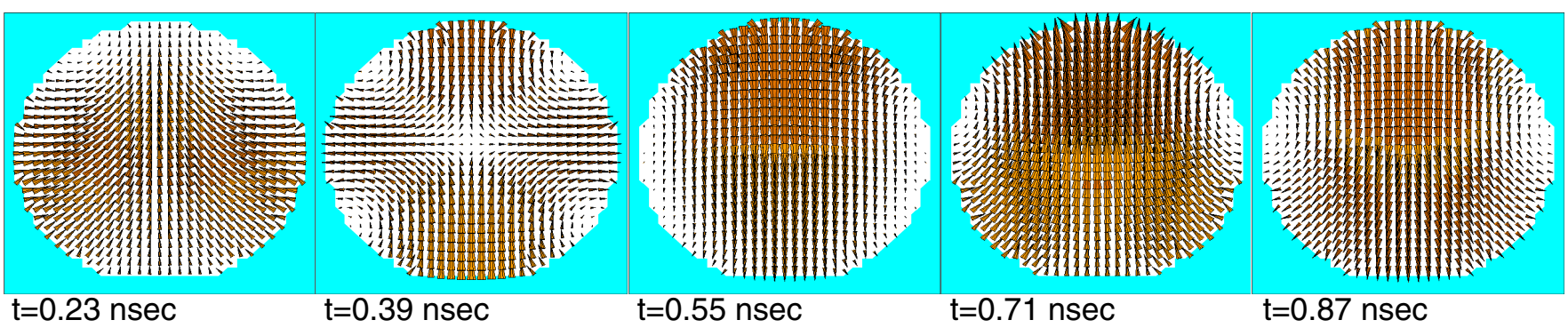

FIG. 8. (Color) Electric field force lines downstream from the collimator at consecutive time periods after the passage of a bunch.

where $f_{0}$ is the resonant frequency of an empty cavity. Additionally for high loss materials the inverse value of the resonance quality factor $Q_{d}$ is almost equal to the loss tangent $\tan \delta$

$$
\frac{1}{Q_{d}} \approx \tan \delta
$$

when the $Q$-value of the empty cavity is high. For the measurement we used a copper rectangular box, which has the same inner sizes as a ceramic tile. The empty copper cavity has a $Q$-value of several thousand. The photo of this box and the tile is shown in Fig. 9. Ceramic tile dimensions are $14.22 \mathrm{~mm} \times 14.58 \mathrm{~mm} \times 5.94 \mathrm{~mm}$. The lowest resonant frequency of the empty cavity is 14.72 GHz. When we place a tile inside the cavity, the resonant frequency drops to $3.27 \mathrm{GHz}$, equivalent to a relative permittivity 20.3. $Q$-measurement resulted in a loss tangent $\tan \delta=0.25$. Ceradyne's table [15] gives dielectric parameters only for three frequencies: 1,8 , and $10 \mathrm{GHz}$. Based on these numbers we approximated its parameters at $3.3 \mathrm{GHz}$ : relative permittivity 26.6 and loss tangent 0.22 . We measured several tiles and found that their parameters do not differ much. Relative permittivity deviates within $\pm 3.5 \%$ and loss tangent $\pm 2.2 \%$, however we found a strong temperature dependence. Results are shown in Fig. 10. The loss tangent drops to almost 0.1 and relative permittivity rises to 25 when the temperature reaches $250^{\circ} \mathrm{F}$. At high $\mathrm{HOM}$ power the working temperature of tiles could be high, so we choose the high temperature

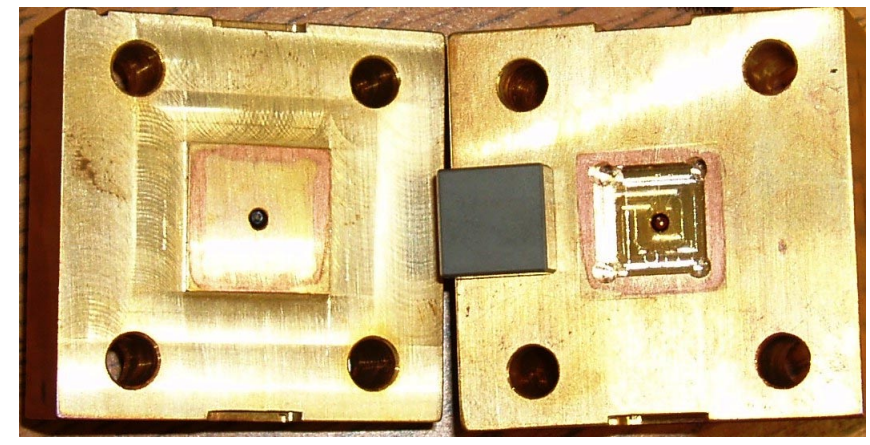

FIG. 9. (Color) Copper cavity with two antennas to measure the dielectric constant and loss tangent of a ceramic tile. parameters of tiles $\epsilon=30, \tan \delta=0.1$ to be used in our computer modeling of the straight absorber.

\section{B. Coupling slots}

We want to damp TM-type waveguide modes with wall currents in the azimuthal direction. By adding long slots parallel to the beam, those image currents will be intersected and strongly disturbed. If a slot has an elongated elliptical shape with semimajor axis $l$ and semiminor axis $d$ and $l \gg d$, then the coupling of the transverse field according to [16] is

$$
k_{\perp} \propto \frac{\pi}{3} \frac{l^{3}}{\ln \frac{4 l}{d}-1}
$$

the coupling of the bunch field is

$$
k_{b} \propto \frac{\pi}{3} l d^{2} .
$$

The ratio between $k_{b}$ and $k_{\perp}$ depends on the ratio of axes $(d / l)^{2}$ and can be made very small. On the other hand, the transverse mode coupling is proportional to the cube of the slot length and can be large enough to transfer out almost all the mode power. For this we will need a slot length of the order of the tube diameter.

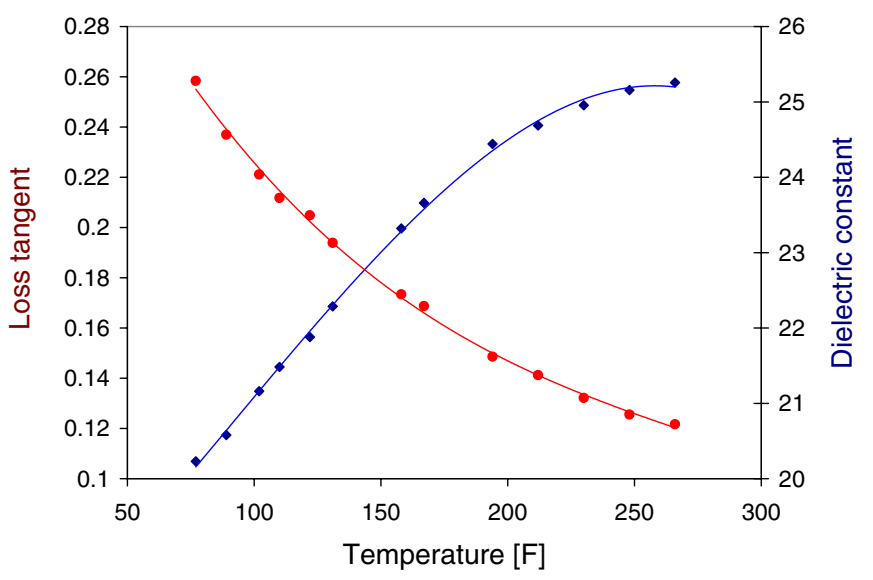

FIG. 10. (Color) Temperature dependence of the dielectric constant (blue) and loss tangent (red) from our measurement. 


\section{Computer modeling of the bellows absorber}

We decided to replace an existing shielded bellows module by a new design in order to place an absorber in the PEP-II low energy ring. In a new design we decrease the bellows convolution length and we use all free space for absorber slots. In this way we managed to find $70 \mathrm{~mm}$ for the absorber. The geometry of the absorber vacuum chamber assembly (one quarter) is depicted in Fig. 11. The vacuum chamber assembly has an annular cavity which houses the bellows assembly and a set of ceramic bricks or tiles (dark gray) separated from the beam chamber by a series of longitudinal coupling slots. This offers a conductive path for monopolelike beam fields while exposing the absorbing material to the transverse fields (dipole and quadrupole). The absorbing tiles are brazed to a copper block with cooling water pipes to extract the heat. The absorber cavity features an extension which exposes the bellows cavity to the absorber. This has the added effect of damping any modes which might be produced by the transverse fields coupling into the bellows through the bellows fingers. For computer simulations we ignore the bellows thin fingers and simplify the bellows convolutions. We use s-parameter analysis to characterize the behavior of an absorber configuration (geometry and material parameters). This capability is included in the MAFIA package [10]. A simulated electromagnetic wave packet of a chosen frequency range propagates through the beam pipe incorporating an absorber structure. To simulate the "halfinfinite waveguides" before and after the configuration, we do a separate calculation for each field mode: monopole, dipole, and quadrupole. In this case we can perfectly match each field model at the "mesh" entry and mesh exit of absorber structure. The amplitudes of the fields are monitored in time at the entry and exit ports of the structure. The length of the simulated configuration is chosen to be several times longer than the wave packet length in order to separate the forward signal and the reflected signal at the entry port. The signal Fourier transform gives the amplitude of the forward wave, reflected wave, and transmitted wave, from which we calculate the transmission

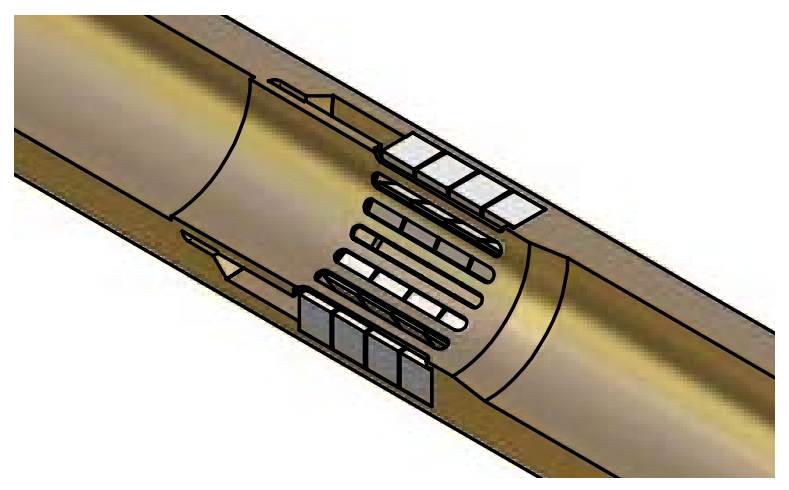

FIG. 11. (Color) The model of the bellows absorber (one quarter). coefficient $s_{21}$ and reflection coefficient $s_{11}$. Fractional power loss (or absorption) is calculated from the energy conservation law:

$$
P=1-\left|s_{11}\right|^{2}-\left|s_{21}\right|^{2}
$$

The computer design process involved variation of slot, absorber, and cavity geometry to optimize absorption characteristics using scattering parameter analysis. We optimized the absorber configuration parameters in order to maximize absorption for dipole and quadrupole modes at the given slot length of $70 \mathrm{~mm}$. At first we study how the slot thickness influences the absorption. To limit the image current density at double the slotless value, we choose the slot width to be the same as metal width. Under this constraint we determine the number of slots for different slot widths. We found that wider slots give more absorption, but also increase the absorption for the monopole (longitudinal) mode, which is undesirable. We choose a compromise for slot width of $6 \mathrm{~mm}$ that gives less than $1 \%$ absorption for the monopole mode. Then we optimized the thickness of tiles and determined that the frequency spectrum follows the tile thickness. Thicker tiles move the spectrum to the low frequency range. The tile thickness of $16.7 \mathrm{~mm}$ gives a more or less flat frequency spectrum in the range 2 to $7 \mathrm{GHz}$. Results of scattering parameter analysis for the optimized absorber configuration are shown in Fig. 12 for three waveguide modes: monopole (top), dipole (middle), and quadrupole (bottom). Average absorption of longitudinal mode is around $0.4 \%$ in the frequency range of 3 to $6 \mathrm{GHz}$. Dipole mode absorption is important since this is the main mode generated by the collimator. In the $2-7 \mathrm{GHz}$ range we can expect on average $31 \%$ of the dipole mode power to be absorbed. Maximum absorption of $53 \%$ is at the frequency of $3.5 \mathrm{GHz}$ over a surrounding $40 \%$ plateau. Absorption of the quadrupole mode is on average $73 \%$ in the frequency range of 3.4$6.5 \mathrm{GHz}$. Minimum frequency is determined by the cutoff frequency of each mode.

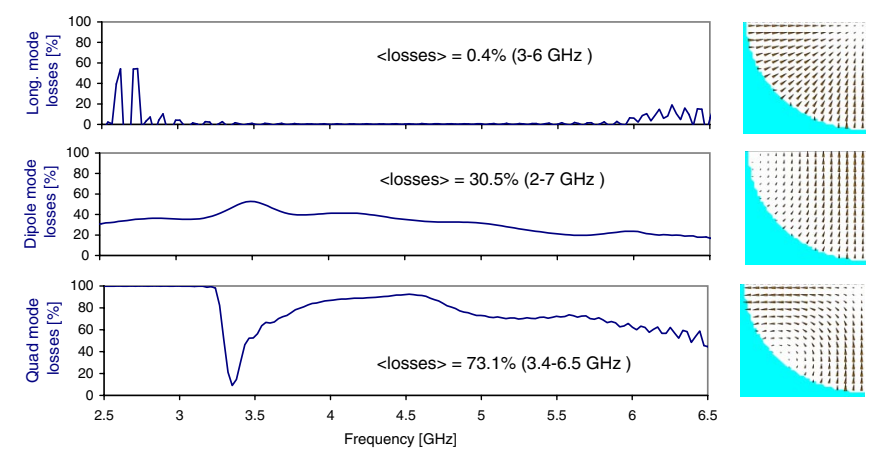

FIG. 12. (Color) Losses for different modes in an absorber. The insets show electric force lines for each mode. 


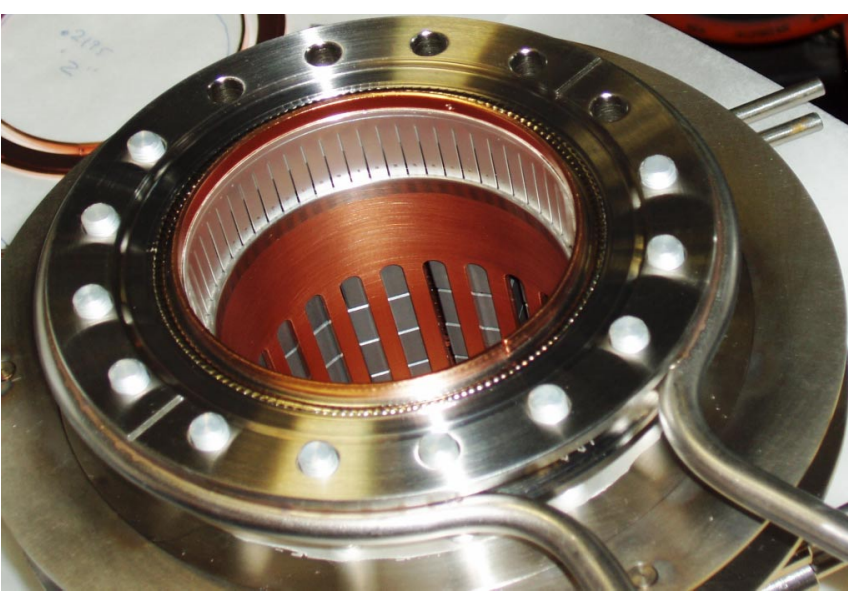

FIG. 13. (Color) Bellows absorber.

\section{Bellows-absorber measurement}

Based on computer modeling a bellows absorber was designed and four absorbers were produced. Figure 13 shows the photo of a completed device just prior to installation. From the photo it is possible to see shielded bellows fingers (white) and coupling absorber slots in the tube (red). Absorbing tiles (gray) are visible behind the coupling slots. Copper water cooling lines remove absorber HOM power. The cooling system was designed to take out more than $10 \mathrm{~kW}$ of HOM power. The first bellows absorber was installed in the low energy ring (LER) in region 4 at the end of a straight and has experienced high current running. The bellows-absorber position in the ring is shown in Fig. 14. Downstream we have a vacuum valve and an arc bellows. This arc bellows has extensive air cooling, and exhibits the maximum temperature rise in the ring. Next we have a quadrupole magnet, a bending magnet, and the first arc pumping chamber, which has an absorber in its antechamber.

To determine the effect of the bellows absorber we measure the temperature of the arc bellows (see photo 14) before and after installation of the bellows absorber. Figure 15 shows the history of the temperature rise in the

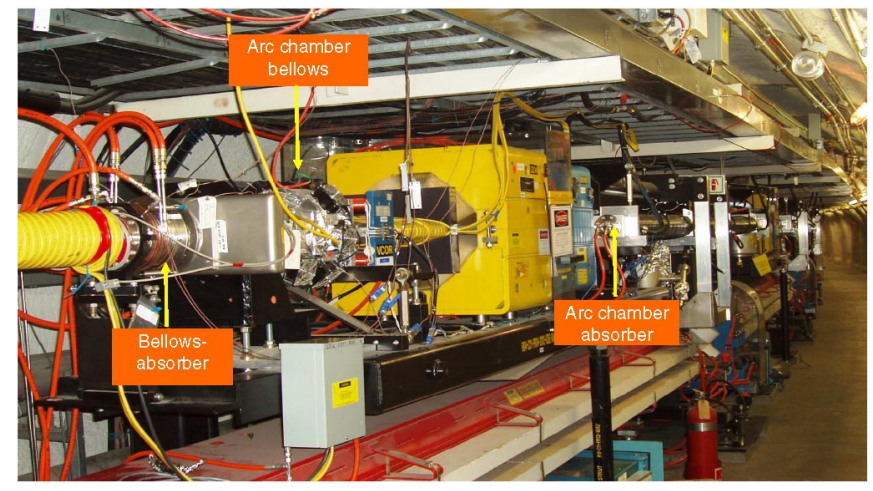

FIG. 14. (Color) SLAC PEP-II tunnel where the bellows absorber is installed.

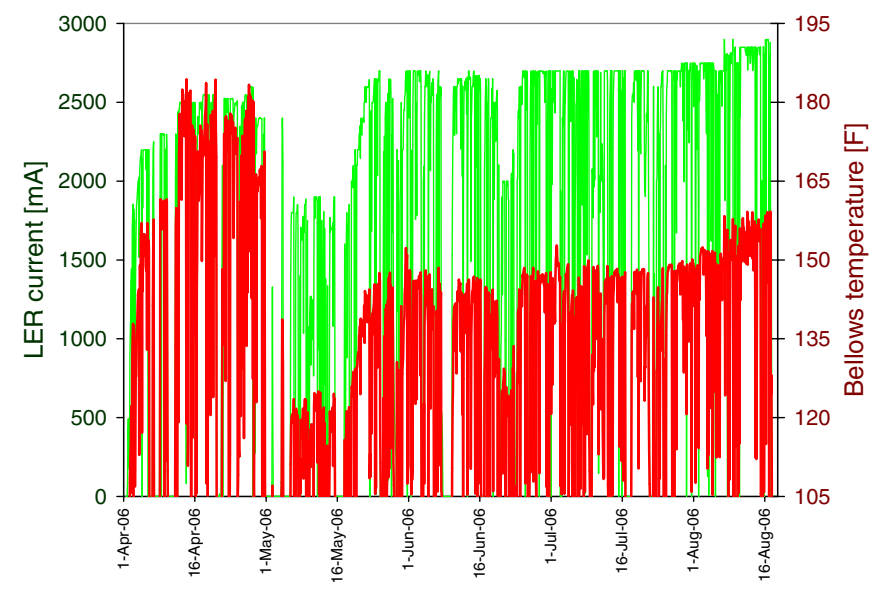

FIG. 15. (Color) Temperature rise in the downstream arc bellows (red) and LER current (green).

arc bellows (red) and LER current (green). After installation of the bellows absorber on May 1, 2006, the arc bellows temperature drops to almost half of the previous value at comparable LER currents. Pumping ports, vacuum valves, and antechambers see a similar reduction.

The efficiency of the bellows absorber was evaluated from the HOM power measurement in the arc chamber absorber. Figure 16 shows the current dependence of the HOM power in this absorber before and after installation of the bellows absorber. We are reminded that the arc chamber absorber takes HOM power only from the vertical collimator.

The HOM power dependence is quadratic with LER current. A fit for the coefficient with data from before and after the installation gives 145 and $82 \mathrm{~W} / \mathrm{mA}^{2}$, respectively. The coefficient ratio gives a $42 \%$ reduction in power. That means that the bellows absorber captures $42 \%$ of the HOM power generated at the vertical collimator. Power in the absorber as a function of the beam current is

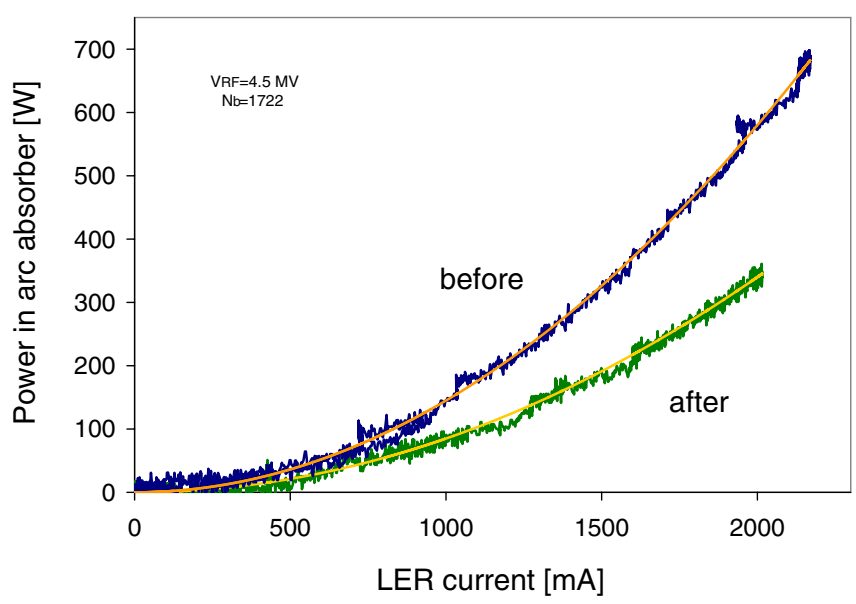

FIG. 16. (Color) HOM power in the downstream arc absorber before (dark blue) and after (green) the installation of the bellows absorber. Orange and yellow lines show a quadratic fit. 


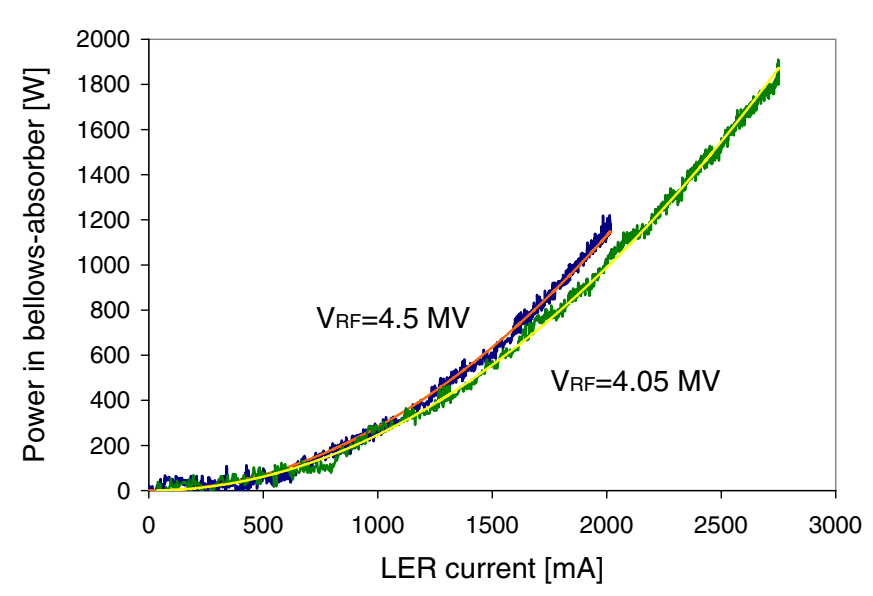

FIG. 17. (Color) Power in the bellows absorber for different rf voltages.

shown in Fig. 17 for two different rf voltages. For the LER current of $2.4 \mathrm{~A}$, the HOM power captured in the bellows absorber reaches $1.45 \mathrm{KW}$. Knowing the efficiency of the absorber, we can estimate the HOM power coming from the vertical collimator at this current

$$
P_{\mathrm{vc}}=1.45 \mathrm{~kW} / 0.42=3.45 \mathrm{~kW} .
$$

This number is very close to the result of wakefield calculation presented in Sec. II.

After successful results with the first bellows absorber, we installed two other absorbers in the same straight section of region 4 but downstream to two horizontal collimators. An additional bellows absorber was installed in the straight section of region 10 .

\section{HIGH-EFFICIENCY ABSORBER FOR THE INTERACTION REGION}

As we discussed earlier, the coupling of transverse fields and, respectively, the efficiency of the HOM absorber strongly depends on the length of the coupling slots. Can we design a more efficient absorber with almost full absorption by increasing the slot length? We study this possibility for an absorber which we plan to install in the interaction region (IR), where we have available space. Unlike the straight section chamber in region 4, the IR vacuum chamber has an octagonal shape. Now the vertical and horizontal polarization of transverse modes are different and are separated in frequency. Different polarizations may have different absorption. We found that this difference can be partially compensated by placing the thicker absorbing ceramic tiles at the shorter sides. Figure 18 shows the geometry of the IR absorber (one quarter). The horizontal size of the chamber is $88.4 \mathrm{~mm}$ and the vertical size is $51 \mathrm{~mm}$. The optimized value of the slot length is around $23 \mathrm{~cm}$, the slot width is $3.4 \mathrm{~mm}$. The absorber tile thickness is $6 \mathrm{~mm}$ at the long side and $12.7 \mathrm{~mm}$ at the short side. Absorption for the longitudinal mode, two polariza-

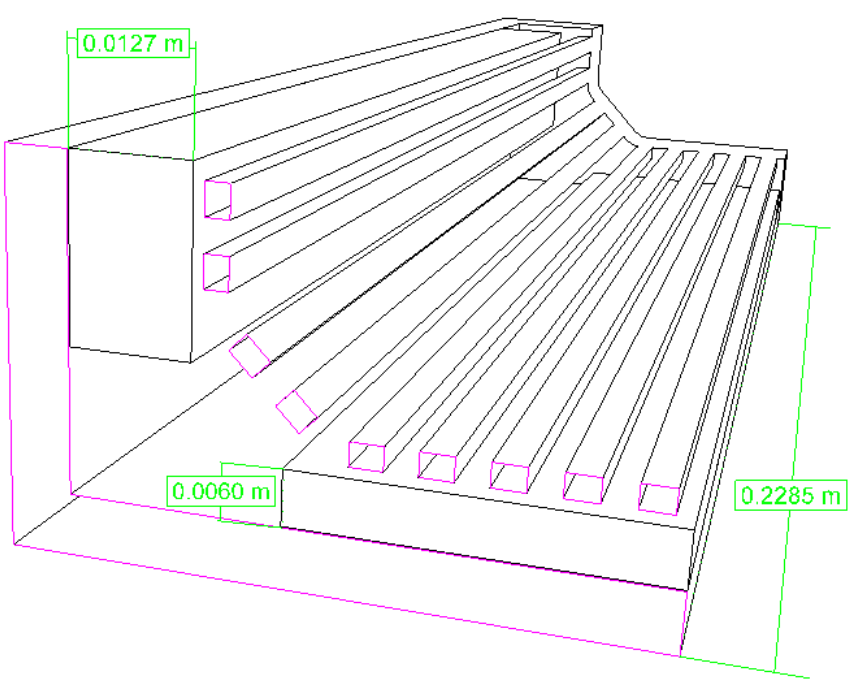

FIG. 18. (Color) IR absorber. One quarter.
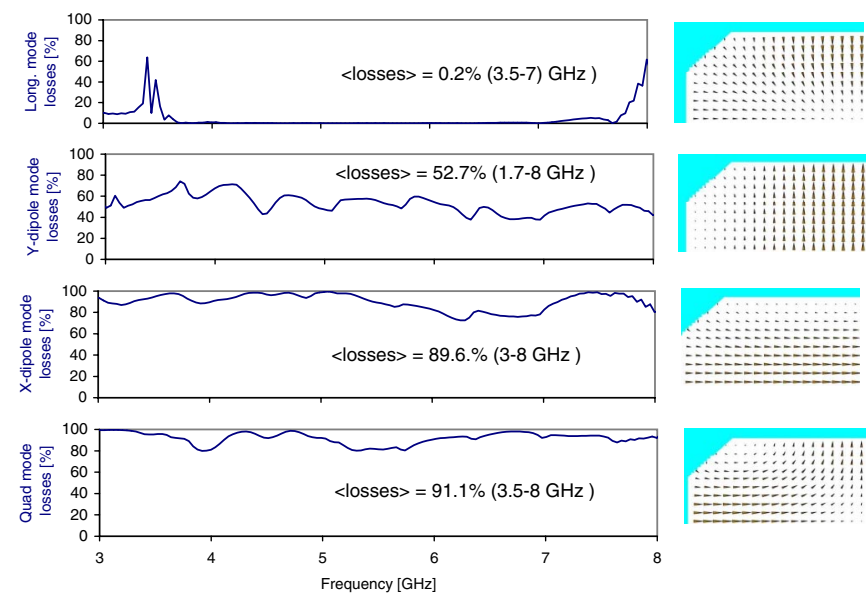

FIG. 19. (Color) Losses of different modes in the IR absorber.

tions of the dipole mode, and quadrupole mode are shown in Fig. 19. In this device we managed to achieve almost 90\% absorption of the dipole mode with horizontal polarization in the frequency range of $3-8 \mathrm{GHz}$ and more than $50 \%$ absorption of the dipole mode with vertical polarization in the frequency range of $1.7-8 \mathrm{GHz}$. The absorption of the quadrupole mode is on average of $73 \%$ in the frequency range of $3.5-8 \mathrm{GHz}$. We keep the average absorption of the longitudinal mode at the level of $0.2 \%$ in the frequency range of 3.5 to $8 \mathrm{GHz}$. This absorber was mechanically designed and fabricated. It is already installed in the ring and we will soon measure HOM power in this absorber. The cooling system can take out up to $30 \mathrm{~kW}$ power.

\section{CONCLUSION}

We designed a new absorbing device for damping transverse wakefields. It is built in a straight section of PEP-II 
LER demonstrating the capability to remove and damp undesirable dipole and quadrupole propagating higher order modes produced at an upstream collimator with minimal impedance to the beam. As a result HOM contribution to beam line heating is reduced without impacting beam stability, allowing higher currents for increased luminosity.

\section{ACKNOWLEDGMENTS}

We wish to acknowledge M. Kosovski, S. DeBarger, N. Kurita, and N. Reeck for the mechanical design of the absorber and the PEP-II team for their help and fruitful discussions. This work was supported by Department of Energy Contract No. DE-AC02-76SF00515.

[1] J. Seeman, J. Browne, Y. Cai, W. S. Colocho, F.-J. Decker, M.H. Donald, S. Ecklund, R. A. Erickson, A. S. Fisher, J. D. Fox, S. A. Heifets, R. H. Iverson, A. Kulikov, A. Novokhatski, V. Pacak, M. T. F. Pivi, C. H. Rivetta, M. C. Ross, P. Schuh, K. G. Sonnad, M. Stanek, M. K. Sullivan, P. Tenenbaum, D. Teytelman, J. L. Turner, D. Van Winkle, M. Weaver, U. Wienands, W. Wittmer, M. Woodley, Y. T. Yan, G. Yocky, M.E. Biagini, and W. Kozanecki, in Proceedings of EPAC'2006, Edinburgh, Scotland.

[2] A. Novokhatski, S. DeBarger, F.-J. Decker, A. Kulikov, J. Langton, M. Petree, J. Seeman, and M. Sullivan, in Proceedings of EPAC 2004, Lucerne, Switzerland, p. 854.

[3] A. Novokhatski, in Proceedings of 2005 Particle Accelerator Conference, Knoxville, Tennessee, p. 289.
[4] T. Tajima, K. Akai, E. Ezura, T. Furuya, K. Hosoyama, and S. Mitsunobu, in Proceedings of 1999 Particle Accelerator Conference, New York, p. 440.

[5] S. Belomestnykh, G. Flynn, W. Hartung, J. Kirchgessner, D. Moffat, H. Muller, H. Padamsee, M. Pisharody, and V. Veshcherevich, Cornell SRF report 950905-12, 1995.

[6] H. Hahn, A. Burrill, R. Calaga, D. Kayran, and Y. Zhao, Physica C (Amsterdam) 441, 239 (2006).

[7] Y. Suetsugu, T. Kageyama, Y. Takeuchi, and K. Shibata, Nucl. Instrum. Methods Phys. Res., Sect. A 533, 295 (2004).

[8] S. Weathersby, M. Kosovski, N. Kurita, A. Novokhatski, and J. Seeman, in Proceedings of the 2005 Particle Accelerator Conference, Knoxville, Tennessee, p. 2173.

[9] S. Weathersby, M. Kosovski, N. Kurita, A. Novokhatski, and J. Seeman, in Proceedings of EPAC'2006, Edinburgh, Scotland

[10] The MAFIA Collaboration, "User Guide" CST GmbH, Darmstadt.

[11] A. Novokhatski, SLAC-PUB-10792, 2004.

[12] S. Heifets, G. Lamberston, and C.-K. Ng, SLAC-AP-122, 1999.

[13] S. Kar and M. Leung, CBP Technical Note \#190, 1999.

[14] R. Pendleton, K. Ko, C. Ng, M. Meubauer, and R. Rimer, in Proceedings of 1995 Particle Accelerator Conference, Dallas, Texas, p. 1800.

[15] Properties of Ceradyne's Advanced Technical Ceramics for Microwave Applications, http://www.ceradyne.com.

[16] C. G. Montgomery et al., M.I.T. Radiation Laboratory Series (McGraw-Hill, New York, 1948), Vol. 8. 